Case Report

\title{
FRONTAL SINUS M UCOCELE FOLLOWING A TRIVIAL FACIAL TRAUMA, PRESENTING AS A PYOCELE- A CASE REPORT
}

\section{Satheesh Kumar Bhandary ${ }^{1}$, Vadisha Srinivas Bhat ${ }^{2} \&$ Rishi Ajay Khanna ${ }^{3}$.}

${ }^{1}$ Professor of ENT, ${ }^{2}$ Associate Professor, ${ }^{3}$ Resident, Department of ENT, K.S. Hegde Medical Academy, Nitte University, Deralakatte, Mangalore - 575018

Correspondence:

Satheesh Kumar Bhandary

Dean, KSHEMA, Professor of ENT, K.S. Hegde M edical Academy, Nitte University, Deralakatte, M angalore - 575018 Mobile : +919845130517 E-mail : sakubaraj@yahoo.com

\begin{abstract}
:
M ucocele of the praranasal sinus is a slowly expanding epithelial-lined lesion containing inspissated mucus that fills the sinus cavity. It can erode bone and therefore may extend intraorbitally or intracranially. M ucocele develop when the mucous outflow from a sinus is obstructed. When a mucocele becomes infected, it is called a pyocele. Here we are presenting a case of frontal sinus mucocele developed following a trivial facial trauma, which later presented a pyocele. Diagnosis was confirmed with CT scan and the pyocele was treated with endoscopic marsupialization.
\end{abstract}

Keywords: Mucocele; Pyocele; Frontal sinus; Facial trauma

\section{Introduction:}

Mucoceles of paranasal sinus are chronic expanding lesions filled with sterile mucus. The accumulation of mucus within the sinus develop when the discharge of mucous from this sinus is obstructed. ${ }^{1}$ The obstruction to the outflow can be due to various causes like chronic inflammation, post surgery, radiotherapy, trauma and tumors. ${ }^{2}$ M ucoceles develop most commonly in the frontal sinus. Ethmoidal sinuses are the next common sinus to be involved. They are uncommon in maxillary and sphenoid sinuses. Mucoceles of the frontal and ethmoidal sinuses present as a slowly expanding painless mass lesion, over the medial aspect of the upper eyelid. They can cause lateral displacement and proptosis of the corresponding eyeball. It can also cause mechanical ptosis. When a mucocele become infected, it is called pyocele, and present with pain and other signs of inflammation. Computed tomography scan of the paranasal sinuses in coronal and

\begin{tabular}{|c|}
\hline Access this article online \\
\hline Quick Response Code \\
\hline
\end{tabular}
axial view is the gold standard investigation for confirmation and diagnosis and to know the extent of the lesion. Surgery is the treatment of choice and can be done either by external or endoscopic approach. Endoscopic marsupialization is a safe and effective approach with less postoperative complications. ${ }^{2}$ Here we report a case of 63 year old male with frontal sinus mucocele developed following a trivial facial trauma, which later became a pyocele and managed byendoscopic marsupialization.

\section{Case Report :}

A 63 year old male presented with the history of a slowly progressive painless swelling over the medial aspect of the left upper eyelid since 2 years. He gave a history of a trivial trauma over the left side of the nose before two years. Since one week he developed pain over the swelling and restriction of the left eye movement. The patient was initially evaluated by the ophthalmologists and found to have mechanical ptosis of left upper eyelid; the eyeball was pushed outwards and downwards ( Fig 1). His eye movements were normal and full range in both the eyes and vision was normal. He was then referred to the department of ENT for further evaluation. On examination there was a soft, tender swelling of $2 \mathrm{~cm} \times 2.5 \mathrm{~cm}$ over the medial aspect of left upper eyelid the margins of which was not well defined. On deep palpation there was a defect of about $0.5 \mathrm{~cm} \times 0.5 \mathrm{~cm}$ noticed on the roof of the left orbit. He had deviated nasal septum to right. Rest of the nasal cavity was normal on anterior rhinoscopy. Diagnostic nasal endoscopy revealed polypoidal left uncinate process. CT 
scan of the paranasal sinus showed homogenous soft tissue opacity in the left frontal sinus, with extension into left orbit with erosion of the posterior table of the left frontal sinus. There was destruction of the left supraorbital ridge (Fig 2, 3). Radiological features confirmed the clinical diagnosis of frontal sinus pyocele.

Endoscopic marsupialization of the left frontal sinus pyocele was done under general anesthesia. About $20 \mathrm{cc}$ pus was drained from the left frontal sinus (Fig 4). Postoperative period was uneventful. Endoscopic cleaning was done after one week. During this period his eye became normal (Fig 5). He is under regular follow up and is symptom free during three months follow up period.

\section{Discussion :}

Mucocele of the frontal sinus was first described by Dezeimeris in $1725^{1}$. Rollet coined the term mucocele to describe these conditions. ${ }^{3}$ Bockmuhl et al identified the most common etiology of frontal mucocele as previous paranasal sinus surgery for chronic rhinosinusitis. ${ }^{2}$ They found this history in $66 \%$ of patients. In their study $17 \%$ of mucoceles developed spontaneously and history of trauma was found in $14 \%$ cases. The patient in our report did not have any sinus related complaints in the past. He had a history of a trivial trauma to the left side of the nose corresponding to the onset of mucocele.

The complaints of patients with mucocele of the frontal sinus depend on the location and extent of the lesion. If the mucocele is confined within the sinus walls, they present with frontal headache, which is the most common symptom in such cases. ${ }^{4}$ M ucocele of the frontal sinus can extend into the orbit, adjacent sinuses or into the nasal cavity. Destruction of the orbital roof and extension into the orbit lead to proptosis and diplopia, occasionally may compress the optic nerve leading to loss of vision. ${ }^{5}$ Posterior table of the frontal sinus may be destroyed by the mucocele due to osteolysis resulting in a communication between the frontal sinus and extradural space. Since the dura can withstand the pressure exerted by the mucocele, the extradural extension may go unnoticed till imaging is performed. Intradural extension can cause meningoencephalitis, pneumocephalus and CSF leak. ${ }^{6}$ Our patient had extension of the mucocele into the orbit resulting in proptosis, but erosion of the posterior table of the frontal sinus and extension into the extradural space was detected only after the CT scan was performed. When a mucocele become infected, it becomes a pyocele and gives rise to the symptoms and signs of inflammation. Our patient had a history of pain in the frontal region developing before one week of his presentation, though he had a swelling for last two years.

Computed tomography (CT) scan is currently the diagnostic method of choice for mucoceles. Coronal and Axial views with three dimensional reconstruction will help the clinician in knowing the extent of the pathology and planning the management. Bone erosions are better detected with CT scans and allows the surgeon to be aware of the landmarks during endoscopic sinus surgery.

Surgery is the treatment of choice in frontal mucoceles. The approach depends on the extent of the mucocele. Since most of the frontal sinus mucocels are located medially, they can be treated by endoscopic marsupialization. Stripping of the mucocele lining is not necessary, because the epithelium return to normal postoperatively. The laterally located frontal mucoceles are difficult to approach endoscopically and may have to be managed by external approach. ${ }^{2}$ Our patient underwent endoscopic marsupialization and the pyocele was drained endoscopically. His eye became normal in the postoperative period and is symptom free during the 3 monthsfollow up period.

To conclude, frontal sinus mucocele can follow a trivial trauma to the face which results in obstruction of drainage of the sinus. Endoscopic marsupialization is a safe and effective approach for management of Frontal sinus mucocele irrespective of etiology. 


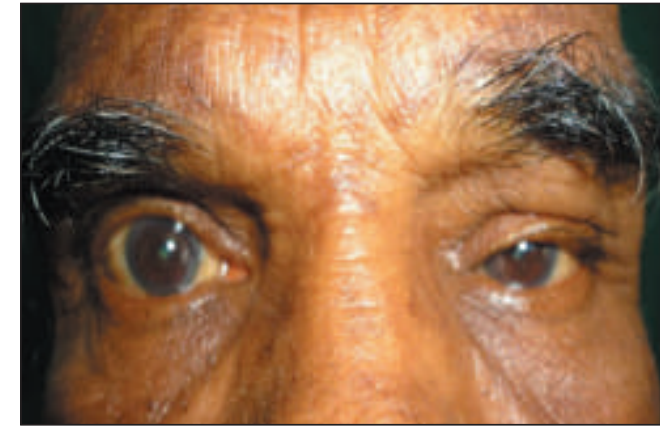

Fig 1 : Pre op photograph- swelling in medial aspect of left upper eyelid with eyeball pushed outwards and downwards

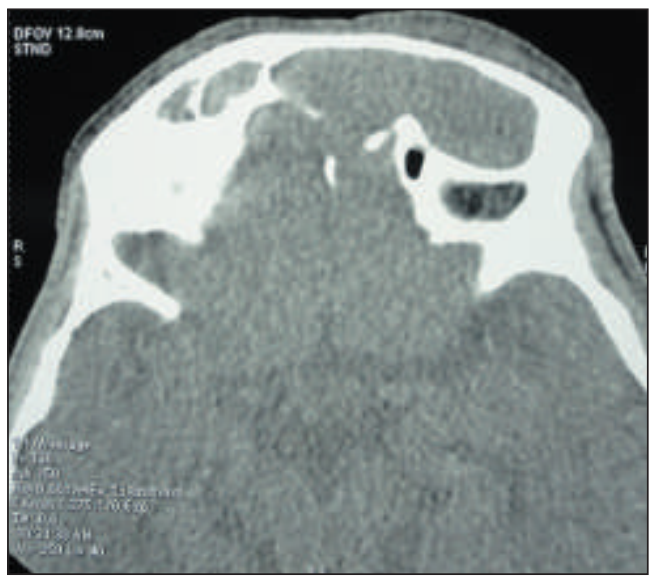

Fig 3: CT scan of PNSAxial view showing erosion of the posterior table of the left frontal sinus

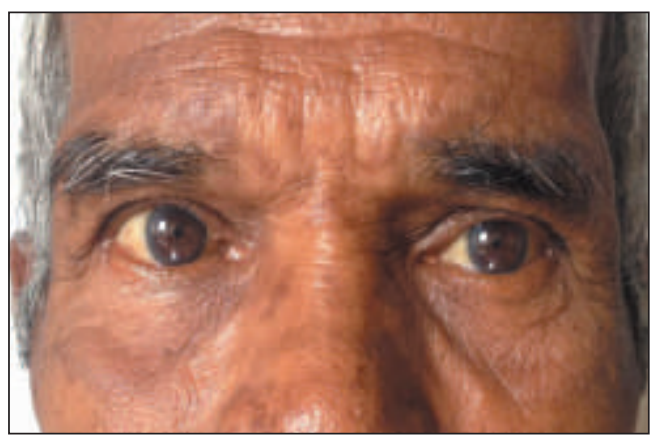

Fig 5 : Post op photograph- Left eye became normal

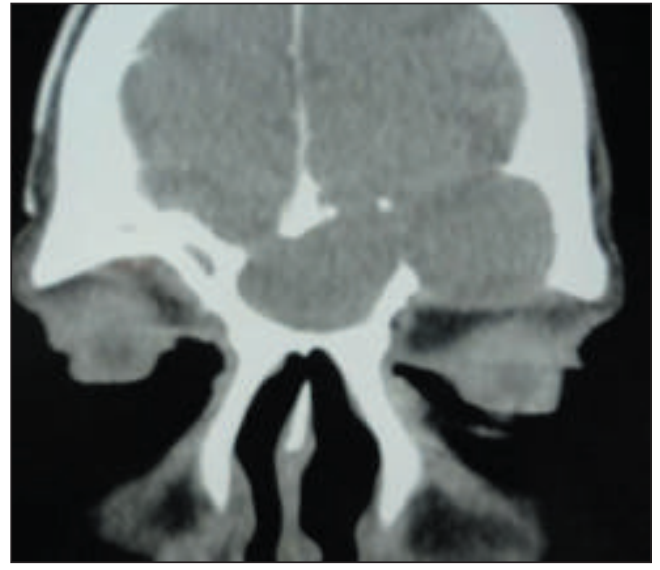

Fig 2 : CT scan of PNS coronal view showing Mucocele in Left Frontal sinus with Orbital extension

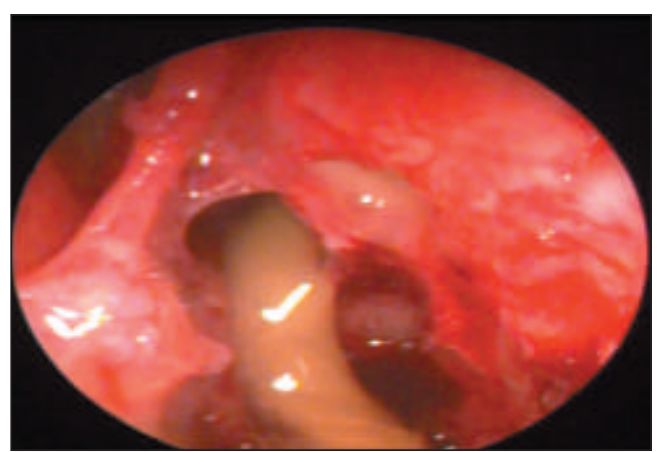

Fig 4 : Endoscopic M arsupialization of pyocele - Pus drainingfrom Frontal sinus

\section{References:}

1. ConstantinidisJ, Steinhart H, Schwerdtfeger K, ZenkJ, Iro H. Therapy of invasive mucoceles of the frontal sinus. Rhinology (2001). 39: 33-38

2. Bockmuhl U, Kratzsch B, Benda K, Draf W. Surgery for paranasal sinus mucoceles: Efficacy of endonasal M icro- endoscopic management and long-term results of 185 patients. Rhinology (2006). 44: 62-67

3. Rollet M. Mucocele de l'angle superointerne des orbites. Lyon Med (1896). 81:573-576

4. Bordley JE, Bosley WR. Mucoceles of the frontal sinus: causes and treatment. Ann Otol (1973). 82:696-702.

5. Fujitani T, Takahashi T, Asai T. Optic nerve disturbance caused by frontal and fronto-ethmoidal mucopyoceles. Arch Otolaryngol (1984). 110:267-269

6. Koike $Y$, Tokoro K, Chiba Y, Susuki S, Murai M, Ito H. Intracranial extension of paranasal sinus mucocele: two case reports. Surg Neurol (1996). 45:44-48. 